\title{
DEPTOR is a direct p53 target that suppresses cell growth and chemosensitivity
}

\author{
Danrui Cui ${ }^{1,2}$, Xiaoqing Dai ${ }^{1,2}$, Longyuan Gong ${ }^{1,2}$, Xiaoyu Chen ${ }^{1,2}$, Linchen Wang ${ }^{1,2}$, Xiufang Xiong ${ }^{2,3}$ and \\ Yongchao Zhao ${ }^{1,2}$
}

\begin{abstract}
DEP-domain containing mTOR-interacting protein (DEPTOR), a natural mTOR inhibitor, has essential roles in several processes, including cell growth, metabolism, apoptosis, and immunity. DEPTOR expression has been shown to be diversely controlled at transcriptional levels in cell- and context-specific manners. However, whether there is a general mechanism for the regulation of DEPTOR expression remains largely unknown. Here, we report that DEPTOR is a downstream target of the tumor suppressor, p53, whose activity is positively correlated with DEPTOR expression both in vitro in cell cultures and in vivo in mouse tissues. Mechanistically, p53 directly binds to the DEPTOR promoter and transactivates its expression. Depletion of the p53-binding site on the DEPTOR promoter by CRISPR-Cas9 technology decreases DEPTOR expression and promotes cell proliferation and survival by activating AKT signaling. Importantly, inhibition of AKT by small molecular inhibitors or genetic knockdown abrogates the induction of cell growth and survival induced by deletion of the p53-binding region on the DEPTOR promoter. Furthermore, p53, upon activation by the genotoxic agent doxorubicin, induces DEPTOR expression, leading to cancer cell resistance to doxorubicin. Together, DEPTOR is a direct p53 downstream target and contributes to p53-mediated inhibition of cell proliferation, survival, and chemosensitivity.
\end{abstract}

\section{Introduction}

Mammalian target of rapamycin (mTOR), an evolutionarily conserved serine/threonine protein kinase, has pivotal roles in the coordination of cell responses to various stimuli and serves as a central regulator of cell proliferation, survival, and autophagy ${ }^{1-3}$. In mammalian cells, mTOR forms two distinct complexes, mTORC1 (consisting of mTOR, raptor, PRAS40, and GBL) and mTORC2 (consisting of mTOR, rictor, $\mathrm{mSin} 1$, protor, and G(BL). It is well known that mTORC1 is mainly involved in the regulation of protein translation, cell growth, and

\footnotetext{
Correspondence: Yongchao Zhao (yongchao@zju.edu.cn)

${ }^{1}$ Key Laboratory of Combined Multi-Organ Transplantation, Ministry of Public Health, the First Affiliated Hospital, Zhejiang University School of Medicine, Hangzhou, China

${ }^{2}$ Institute of Translational Medicine, Zhejiang University School of Medicine, Hangzhou, China

Full list of author information is available at the end of the article

These authors contributed equally: Danrui Cui, Xiaoqing Dai

Edited by M. Agostini
}

proliferation by phosphorylating S6K1 and 4E-BP1, whereas mTORC2 regulates cell survival by directly phosphorylating and activating $\mathrm{AKT}^{1,2}$. DEP-domain containing mTOR-interacting protein (DEPTOR) inhibits the kinase activity of both mTORC1 and mTORC2 by directly binding $\mathrm{mTOR}^{4}$. Given that mTOR signaling is frequently activated in many human cancers, DEPTOR usually acts as a tumor suppressor, by inhibiting mTOR, to suppress cell growth, and survival via inactivation of $\mathrm{AKT}^{5,6}$. However, in specific cancer types, such as multiple myeloma and T-cell acute lymphoblastic leukemia, DEPTOR exhibits oncogenic properties by activating AKT to alleviate the negative feedback effect of the mTORC1 substrate, S6K1, on IRS1/PI3K signaling ${ }^{7}$. Given that DEPTOR expression is highly cell-specific and context-dependent ${ }^{7}$, its transcriptional regulation likely contributes to the complexity of its roles in tumorigenesis. Thus, it is important to determine whether the expression

\section{(c) The Author(s) 2020}

(c) (i) Open Access This article is licensed under a Creative Commons Attribution 4.0 International License, which permits use, sharing, adaptation, distribution and reproduction cc) in any medium or format, as long as you give appropriate credit to the original author(s) and the source, provide a link to the Creative Commons license, and indicate if changes were made. The images or other third party material in this article are included in the article's Creative Commons license, unless indicated otherwise in a credit line to the material. If material is not included in the article's Creative Commons license and your intended use is not permitted by statutory regulation or exceeds the permitted use, you will need to obtain permission directly from the copyright holder. To view a copy of this license, visit http://creativecommons.org/licenses/by/4.0/. 
of DEPTOR can be regulated under both normal and stressed conditions.

In response to various cellular stresses, including DNA damage, oncogenic activation, hypoxia, and ribosomal stress, the tumor suppressor, p53, is activated to transcriptionally activate or repress the expression of downstream target genes, which play important roles in the regulation of cell cycle arrest, DNA damage response and repair, apoptosis, and senescence, to maintain cellular homeostasis $^{8-11}$. p53, as a transcription factor, specifically recognizes and directly binds to consensus DNA sequences to activate or repress the transcription of downstream target genes, such as $M D M 2^{12}, p 21^{13}$, $N O X A^{14}, R P S 27 L^{15,16}$, and PUMA ${ }^{17}$. The canonical p53binding motif RRRC(A/T)(A/T)GYYY $(\mathrm{N})_{0-13} R R R C(\mathrm{~A} / \mathrm{T})$ (A/T)GYYY (where R represents A or G, Y represents C or $\mathrm{T}$, and $\mathrm{N}$ represents any nucleotide) is usually located near the transcription start site ${ }^{10}$. Accumulating evidence has shown that p53 induces the transcription of several upstream inhibitors of mTORC1, including PTEN, TSC2, and REDD1, resulting in the suppression of mTORC1 activity, which is frequently found to be increased in multiple cancers ${ }^{18,19}$. Thus, it is intriguing to explore the underlying correlation between p53 and mTORC2 activity via DEPTOR, a component and natural inhibitor of the mTORC1 and mTORC2 complexes.

In this study, we demonstrated that p53 directly binds to the DEPTOR promoter and activates its transcription. p53-mediated DEPTOR expression suppressed cell proliferation and survival by inhibiting AKT activity in unstressed conditions. In addition, activation of p53 by genotoxic agents (e.g., doxorubicin) significantly enhanced DEPTOR expression and induced cell resistance to doxorubicin by alleviating the feedback inhibition from S6K1 to IRS1, to activate AKT. Together, we revealed a novel mechanism by which p53 regulates cell proliferation, survival, and chemosensitivity by directly transactivating DEPTOR expression.

\section{Results}

\section{DEPTOR expression is dependent on the presence of p53} in cancer cells and mouse tissues

p53 has an important role in the regulation of mTORC1 activity through the induction of PTEN, TSC2, and $\operatorname{REDD} 1^{18,19}$. However, it is unclear whether p53 can regulate the activity of both mTORC1 and mTORC2 by targeting DEPTOR expression. To explore the interplay between p53 and DEPTOR, we first examined the protein levels of DEPTOR in multiple cancer cell lines with distinct p53 statuses whose transcriptional activity was confirmed by determining the basal and induced levels of endogenous $M D M 2$ and $p 21$, two well-established p53 target genes, upon exposure to ionizing radiation (IR) to activate p53 (Figure S1A and S1B). We found that breast cancer cells
(MCF7 and ZR75-1) and prostate cancer cells (LNCap) harboring wild-type p53 had higher levels of DEPTOR, whereas other cancer cells harboring mutant $\mathrm{p} 53$, including SK-BR3, MDA-MB-231, and DU145 and p53-null cells, such as PC3, had lower or undetectable levels of DEPTOR, indicating a positive correlation between DEPTOR protein levels and p53 activity (Figure S1C). To determine a causal relationship, we silenced the expression of p53 via siRNA oligonucleotides and observed a significant decrease in the protein levels of DEPTOR and MDM2, serving as a positive control, in all the tested cancer cell lines harboring wildtype p53 (Fig. 1a). Notably, the mRNA levels of DEPTOR and $M D M 2$ were downregulated correspondingly to their respective protein levels upon p53 silencing (Fig. 1b). Consistently, both the protein and mRNA levels of DEPTOR were decreased in HCT116 $p 53^{-/-}$colon cancer cells compared with those in paired HCT116 $p 53^{+/+}$cells (Fig. 1c, d). To further demonstrate the positive correlation between DEPTOR expression and p53 activity, we depleted p53 by CRISPR-Cas9 technology in U2OS osteosarcoma cells harboring wild-type p53 and found an obvious decrease in the protein and mRNA levels of DEPTOR in two individual p53-null clones (Fig. 1e, f). Together, these results suggested that p53 positively regulates DEPTOR expression.

More importantly, we found that the protein and mRNA levels of Deptor were lower in the spleen, brain, and kidney from $\operatorname{Trp}_{53} 3^{-1-}$ mice than in those from $\operatorname{Trp}_{5} 3^{+/+}$littermates (Fig. 1g, h), indicating that p53 may regulate DEPTOR expression in vivo under physiological conditions.

\section{p53 directly activates DEPTOR transcription}

We then investigated whether p53 directly activates the transcription of DEPTOR. First, we performed bioinformatics analysis of the DEPTOR promoter and identified three putative p53 consensus binding sites at $-2836 \sim-2817$ (A-DEPTOR), $-2826 \sim-2799$ (B-DEPTOR), and -196 -169 (C-DEPTOR), located upstream of the "start" codon of DEPTOR (Fig. 2a). Then, using a dualluciferase reporter assay, we found that, compared to the pGL3 control, the activity of the luciferase reporter driven by the DEPTOR promoter (DEPTOR-luc), containing all of the three putative p53 consensus binding sites, was increased more than 10-fold in both U2OS and SJSA cells with wild-type p53, whereas DEPTOR-luc activity was strongly suppressed upon p53 depletion (Fig. 2b), indicating p53-dependent transcriptional activation of DEPTOR. To determine the exact p53-binding site in the $D E P T O R$ promoter, we further constructed two luciferase reporters with the deletion of putative p53-binding sites $(\triangle \mathrm{AB}$ and $\Delta \mathrm{C})$. Results showed an obvious decrease in the activity of the luciferase reporter without site $C$ (Fig. 2c), suggesting that the putative p53-binding site $C$, but not 


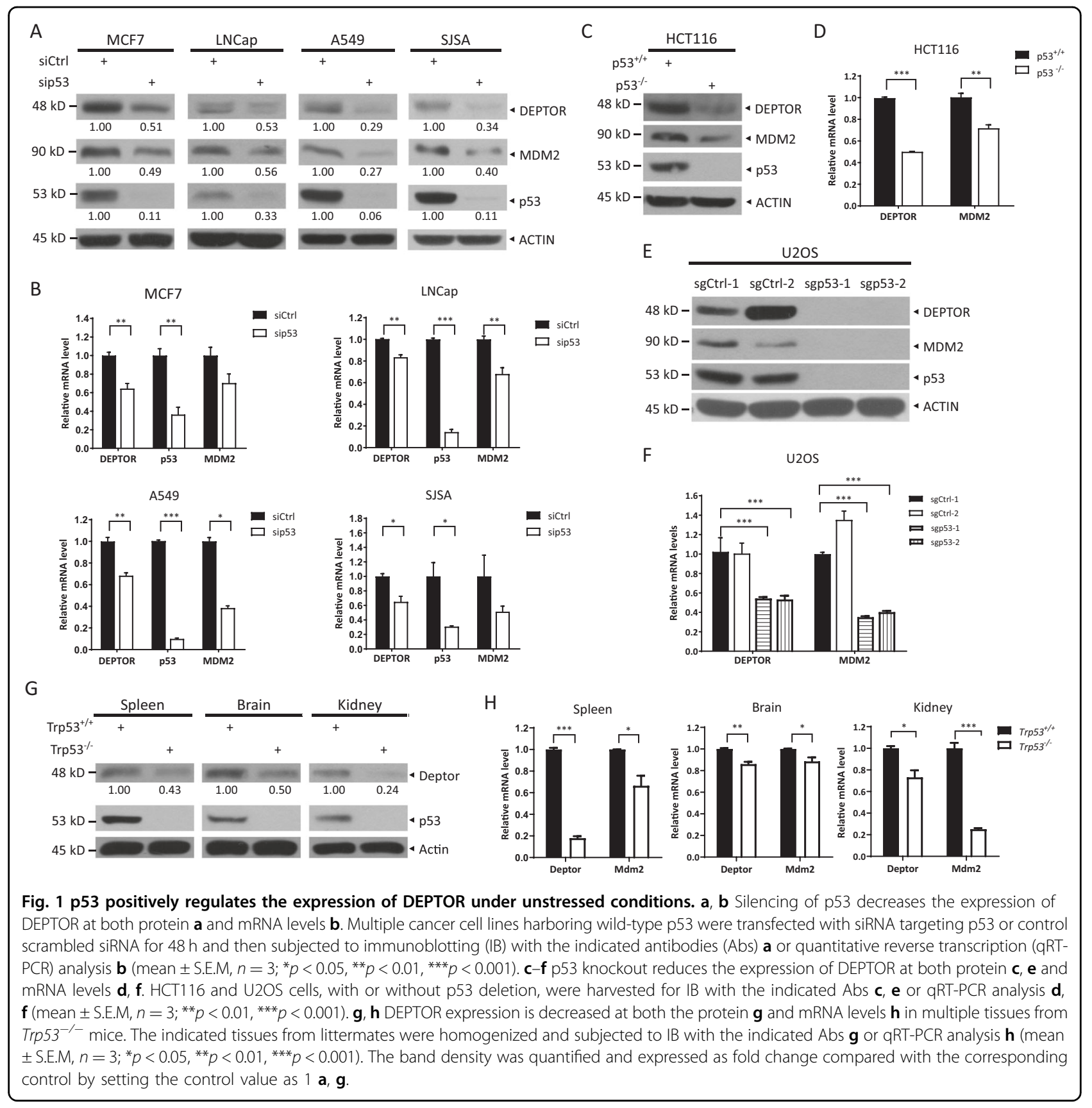

sites $\mathrm{A}$ and $\mathrm{B}$, is important for the activity of the DEPTOR promoter. Moreover, we used CRISPR-Cas9 technology to delete site $\mathrm{C}$ from the DEPTOR promoter on chromosome 8 , without disturbing its "start" codon, to examine whether the putative p53-binding site $\mathrm{C}$ controls DEPTOR expression under physiological conditions. Indeed, both the mRNA and protein levels of DEPTOR were significantly downregulated when site $\mathrm{C}$ was deleted in both U2OS and SJSA cells $(\triangle \mathrm{C}-D E P T O R)$, indicating that site $\mathrm{C}$ may play a role in p53-mediated DEPTOR transcription (Fig. 2d, e). More importantly, we performed chromatin immunoprecipitation (ChIP) assays and detected a physical interaction between the p53 protein and site $\mathrm{C}$ of the DEPTOR promoter, but no interaction between $\mathrm{p} 53$ and sites $\mathrm{A}$ or $\mathrm{B}$. The MDM2 promoter, containing a well-established p53-binding site, was used as a positive control (Fig. 2f, left). And relative fourfold enrichment of the p53-binding site $\mathrm{C}$ on DEPTOR promoter was quantified by qRT-PCR analysis (Fig. 2f, right). Taken together, these results demonstrated that p53 directly binds to site $\mathrm{C}$ of the DEPTOR promoter $(-196 \sim-169)$ and activates its transcription. 


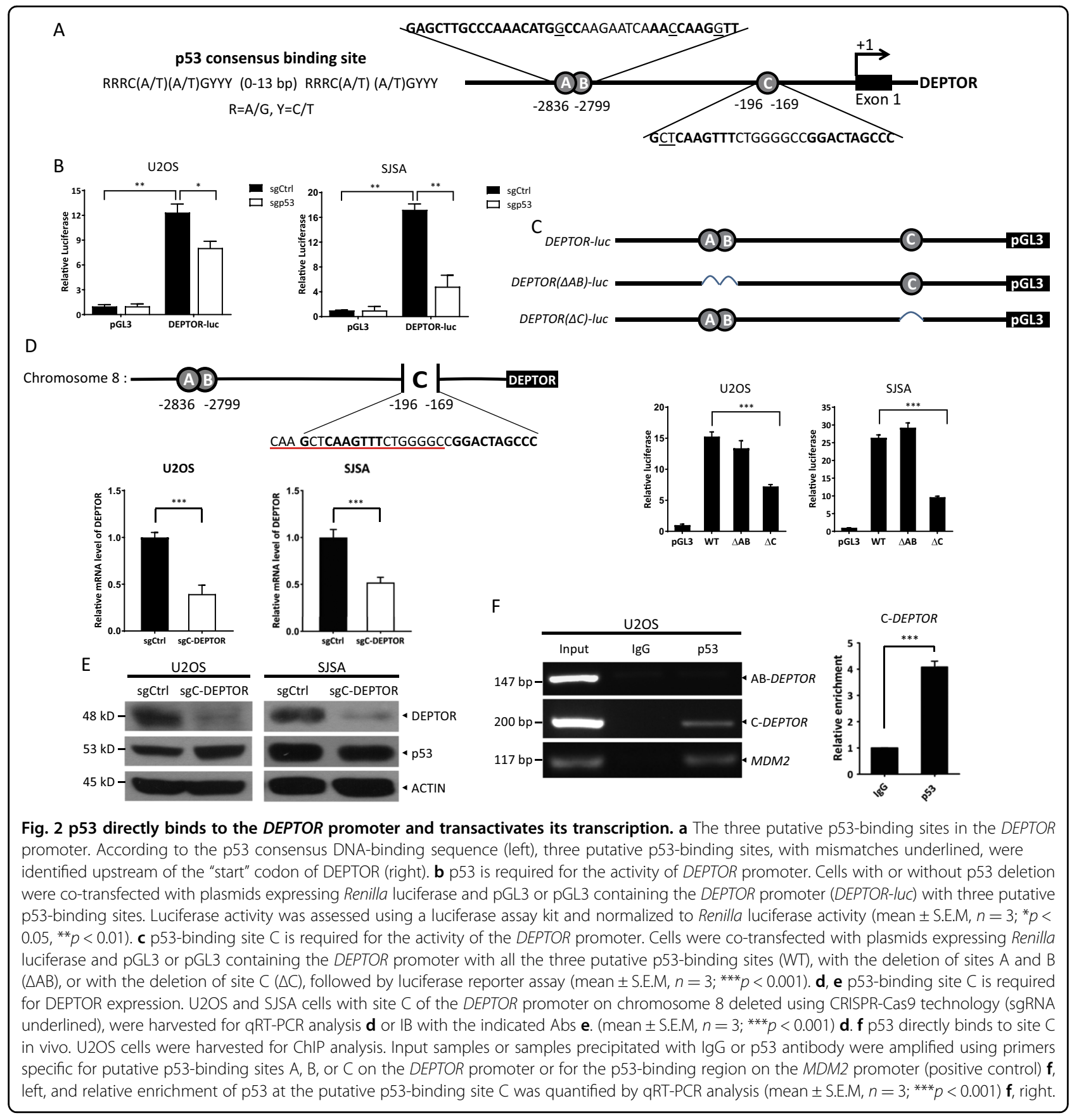

p53 inhibits cell proliferation, survival, and cell cycle progression via the induction of DEPTOR

Next, we evaluated the biological consequences of disturbing p53-mediated DEPTOR expression. The protein levels of DEPTOR were increased more dramatically in HCT116 $p 53^{+/+}$cells than in HCT116 $p 53^{-/-}$cells when grown to a higher density by seeding a great number of cells (Figure S2A) or by increasing the culture time with the same number of cells (Figure S2B). Consistently, the cell density-dependent increase in DEPTOR protein levels was nearly completely abrogated upon depletion of site $C$ in the DEPTOR promoter ( $\triangle \mathrm{C}-D E P T O R)$ (Fig. 3a). Given that DEPTOR is a natural inhibitor of mTOR, which regulates cell proliferation and survival, we hypothesized that cells at a higher confluency express higher levels of DEPTOR transcribed by p53 to suppress mTOR activity and prevent cell overgrowth, as a negative feedback signal to inhibit cell proliferation.

We then performed ATPlite and colony formation assays to examine whether p53-mediated DEPTOR 

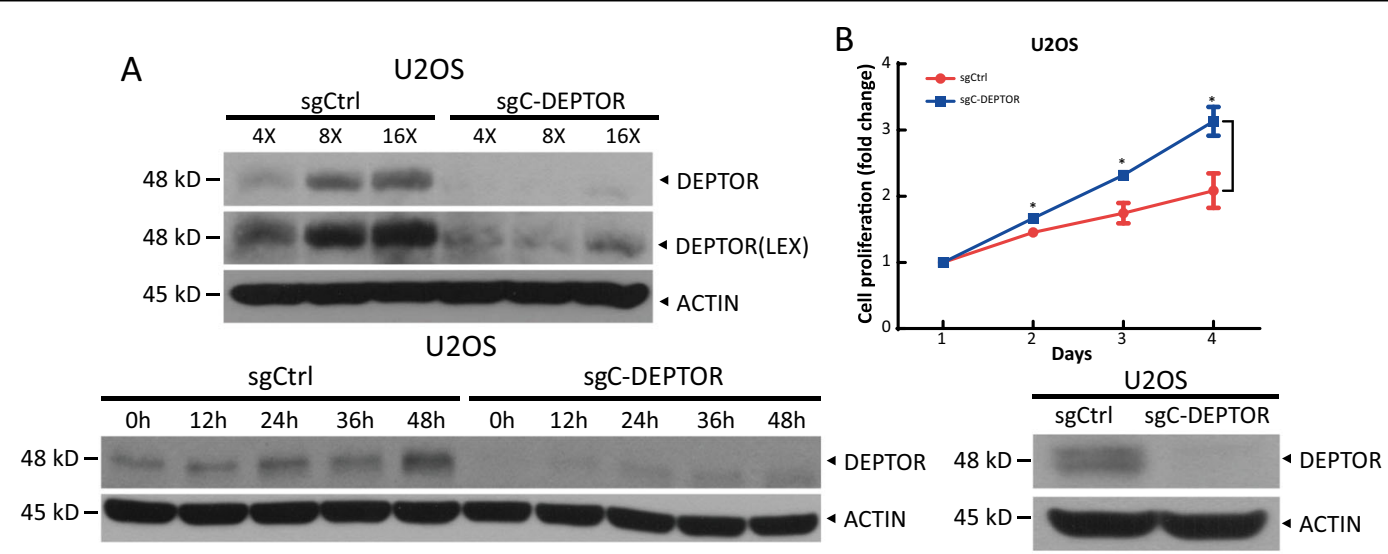

C

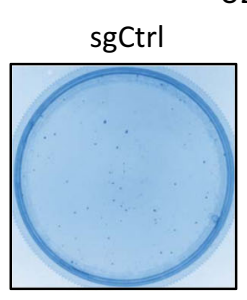

D

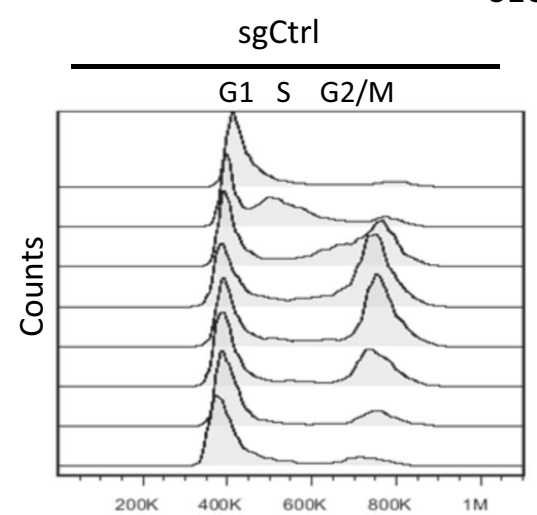

E

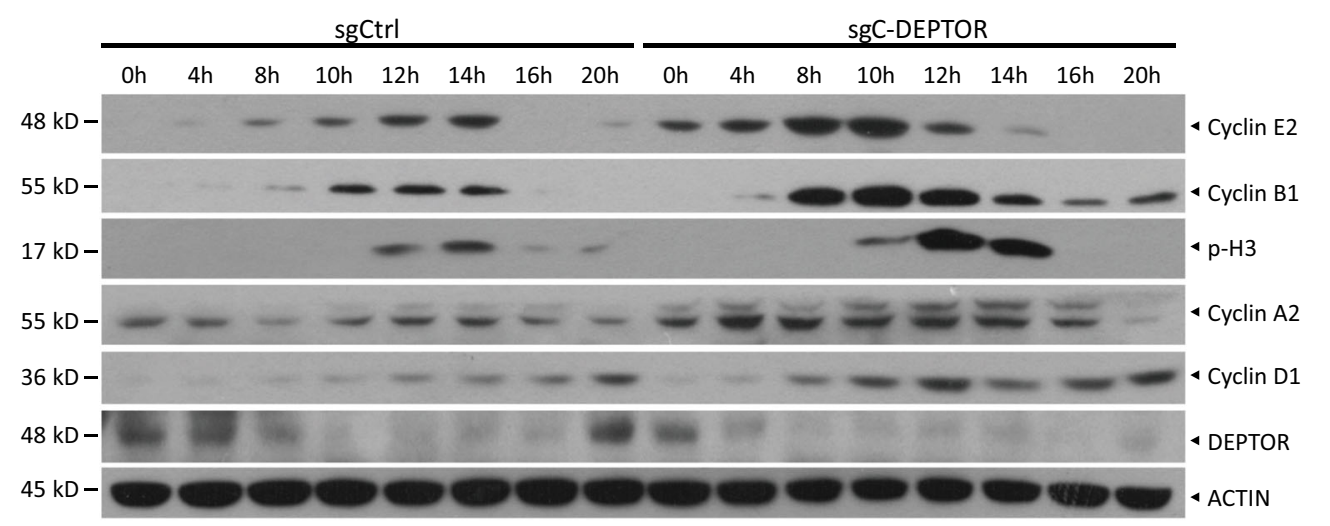

Fig. 3 (See legend on next page.) 
(see figure on previous page)

Fig. 3 p53-mediated DEPTOR expression suppresses cell proliferation, survival, and cell cycle progression. a Deletion of p53-binding site C on the DEPTOR promoter abrogates the increase in DEPTOR expression in a cell density-dependent manner. U2OS cells, with or without the $\mathrm{C}$ site, were seeded at different concentrations $\left(4 \times 10^{5}, 8 \times 10^{5}\right.$, and $\left.16 \times 10^{5}\right)$ in $100 \mathrm{~mm}$ dishes and grown for $24 \mathrm{~h}$ (top) or seeded at the same density and grown for the indicated time periods (bottom). Cells were then harvested and subjected to IB with the indicated Abs. b Deletion of p53-binding site C on the DEPTOR promoter accelerates cell proliferation. Cells with or without site C of the DEPTOR promoter were seeded in triplicate in 96-well plates and grown for various time periods, followed by ATPlite-based cell proliferation assay. Cell proliferation is expressed as the fold change compared with day 1 (mean \pm S.E.M, $n=3$; ${ }^{*} p<0.05$ ). c Deletion of $p 53$-binding site $C$ on the DEPTOR promoter promotes clonogenic survival. Cells with or without site $C$ of the DEPTOR promoter were seeded in triplicate in $60 \mathrm{~mm}$ dishes at 300 cells per dish and incubated for 14 days, followed by staining (left) and colony counting (right) (mean \pm S.E.M, $n=3 ;{ }^{* * *} p<0.001$ ). d, e Deletion of p53-binding site $C$ on the DEPTOR promoter accelerates cell cycle progression. Cells were treated with $2 \mathrm{mM}$ thymidine for $14 \mathrm{~h}$ and then released for $9 \mathrm{~h}$. Next, cells were treated with thymidine for an additional $14 \mathrm{~h}$ and then incubated in fresh medium for different time periods. Cells were then harvested for flow cytometry $\mathbf{d}$ or IB with the indicated Abs e.

expression regulates cell proliferation and survival. Indeed, depletion of site $\mathrm{C}(\triangle \mathrm{C}-D E P T O R)$ significantly promoted cell proliferation (Fig. 3b) and colony formation (Fig. 3c), along with the suppression of DEPTOR expression (Fig. 3b), indicating that DEPTOR expression induced by p53 may suppress cell proliferation and survival. Moreover, we performed a rescue experiment to exclude the possibility of off-target effects due to CRISPRCas9 technology. We reconstituted $\triangle \mathrm{C}-D E P T O R$ cells with ectopically expressed DEPTOR by infecting with a retrovirus expressing FLAG-DEPTOR (Figure S3B) or transfecting with FLAG-tagged DEPTOR plasmid (Figure S3E) and found that both stable and transient expression of exogenous DEPTOR significantly reversed the promotion of cell proliferation (Figure S3A and S3D) and survival (Figure S3C and S3F) induced by depletion of $D E P T O R$ site C. Finally, we synchronized U2OS WT$D E P T O R$ and $\triangle \mathrm{C}-D E P T O R$ cells at the end of G1 phase by double thymidine treatment and then released them back into the cell cycle to evaluate the role of p53mediated DEPTOR expression in cell cycle progression. Flow cytometry results showed that most of the $\triangle \mathrm{C}$ DEPTOR cells entered into the $\mathrm{S}, \mathrm{G} 2 / \mathrm{M}$, and $\mathrm{G} 1$ phases at 4,8 , and $14 \mathrm{~h}$ post release (one cell cycle is $\sim 14 \mathrm{~h}$ ), respectively, whereas the whole-cell cycle of U2OS WTDEPTOR cells was $\sim 20 \mathrm{~h}$ (Fig. $3 \mathrm{~d}$ ), suggesting that the depletion of site $\mathrm{C}$ accelerates cell cycle progression. Immunoblotting analysis of the same cell fractions at each time point largely supported the results of flow cytometry. Specifically, an increase in the levels of cyclin E2, a marker of the G1/S phase, was observed at $4 \mathrm{~h}$ post release in $\triangle \mathrm{C}$ $D E P T O R$ cells, but was delayed to $8 \mathrm{~h}$ in WT-DEPTOR cells. The levels of cyclin B1, which are significantly increased in the G2/M phase, were dramatically increased at $8 \mathrm{~h}$ post release in $\triangle \mathrm{C}-D E P T O R$ cells, but at $10 \mathrm{~h}$ post release in WT-DEPTOR cells. Further, phosphorylation of histone $\mathrm{H} 3$ ( $\mathrm{p}-\mathrm{H} 3)$, as a mitotic marker (M phase), was observed at $10 \mathrm{~h}$ and was diminished at $14 \mathrm{~h}$ post release in $\triangle \mathrm{C}-D E P T O R$ cells, whereas WT-DEPTOR cells began to enter $M$ phase at $12 \mathrm{~h}$ and some cells remained in this phase at $20 \mathrm{~h}$ post release (Fig. 3e). These results demonstrated that disturbing p53-mediated DEPTOR expression accelerates cell cycle progression. Collectively, these results indicated that p53 activated DEPTOR expression to inhibit cell proliferation and survival and delay cell cycle progression.

\section{p53-mediated DEPTOR transcription suppresses cell proliferation and survival by inactivating AKT signaling}

To understand the mechanism by which p53-mediated DEPTOR expression inhibited cell proliferation and survival, we first compared the activity of mTORC1 and mTORC2 in WT-DEPTOR and $\triangle \mathrm{C}-D E P T O R$ U2OS cells. We found that $\triangle \mathrm{C}-D E P T O R$ cells exhibited higher mTORC2 activity. Specifically, after serum starvation for $24 \mathrm{~h}$, the basal level of AKT phosphorylation at Ser 473, reflecting mTORC2 activity, was dramatically higher in $\triangle \mathrm{C}-D E P T O R$ cells than in WT-DEPTOR cells (Fig. 4a, lanes 5 vs 1). After serum stimulation to activate mTORC1 and mTORC2, AKT phosphorylation at Ser 473 increased rapidly and was maintained at high levels for $>8 \mathrm{~h}$ in $\triangle \mathrm{C}-D E P T O R$ cells (Fig. 4a), indicating higher mTORC2 activity after serum stimulation upon deletion of site $\mathrm{C}$ in the DEPTOR promoter. However, the activity of mTORC1, as reflected by S6K1 phosphorylation, was not significantly different between WT-DEPTOR and $\triangle \mathrm{C}$ DEPTOR cells (Fig. 4a). These results suggested that $\mathrm{p} 53$ may induce DEPTOR expression to inhibit AKT activity, leading to the suppression of cell proliferation and survival. To confirm the causal role of AKT activation in the induction of cell proliferation and survival upon depletion of DEPTOR site $\mathrm{C}$, we inactivated AKT using gene knockdown or small molecular inhibitors and found that AKT silencing or MK-2206 (an AKT inhibitor) treatment remarkably inhibited the proliferation of $\triangle \mathrm{C}-D E P T O R$ cells, but had a minor or no effect on the proliferation of WT-DEPTOR cells (Fig. 4b, d). Furthermore, silencing of AKT also abrogated the increase in the survival of $\triangle \mathrm{C}$ DEPTOR cells, as reflected by decreased colony formation (Fig. 4c). Together, our results supported the notion that 


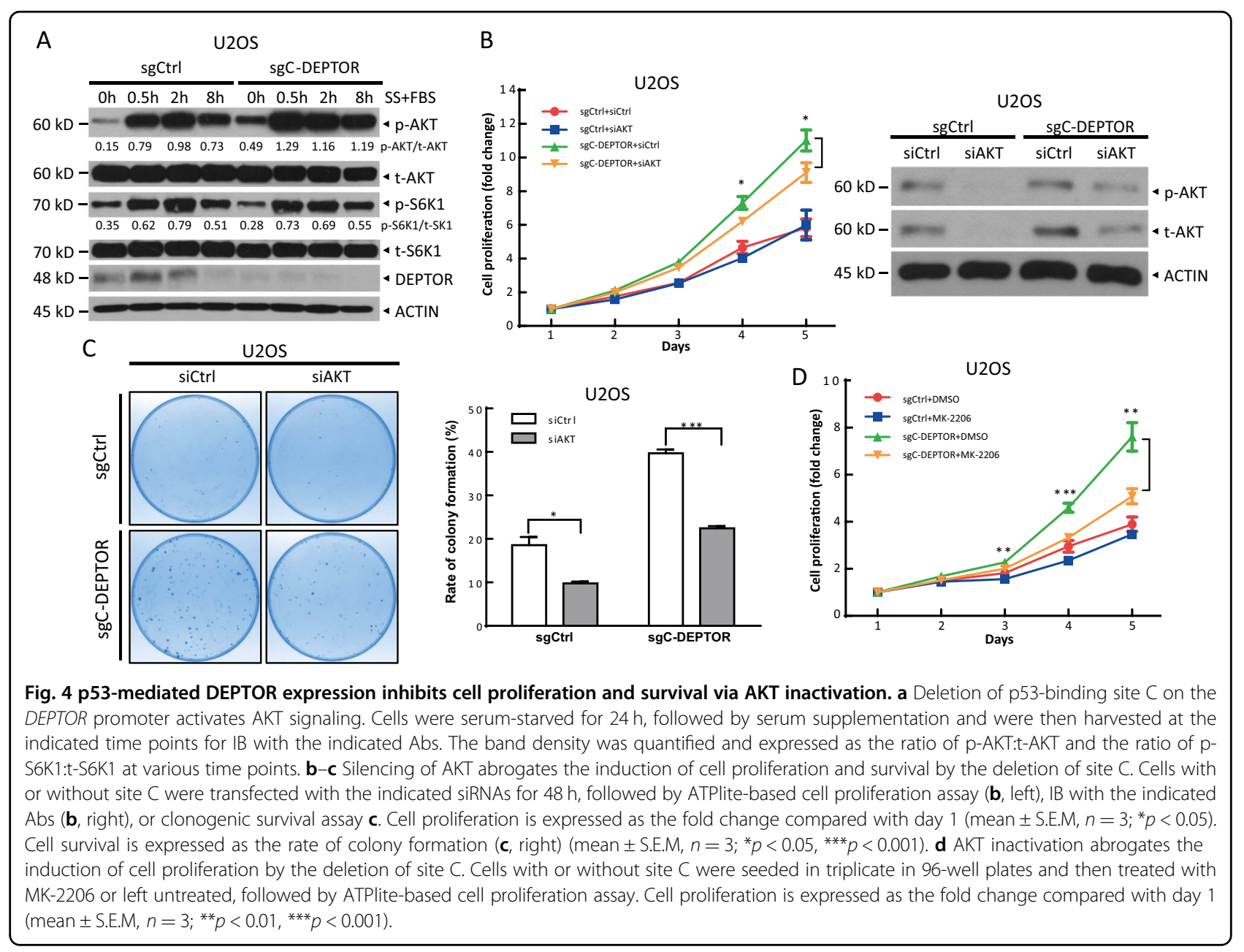

inhibiting p53-mediated DEPTOR expression promoted cell proliferation and survival by activating AKT.

\section{p53-induced DEPTOR expression suppresses cancer cell sensitivity to doxorubicin}

Given that p53 is strikingly activated in response to several stresses, especially DNA damage, we then determined whether $\mathrm{p} 53$ promotes DEPTOR expression upon treatment with different chemotherapeutic agents that trigger DNA damage. Indeed, p53 was activated by several genotoxic agents, including bleomycin, mitomycin $\mathrm{C}$, aphidicolin, hydroxyurea, methotrexate, cyclophosphamide, doxorubicin, teniposide, camptothecin, paclitaxel, cisplatin, and actinomycin D, as well as nutlin-3, an agent that interrupts the interaction between MDM2 and p53 to stabilize p53, in both U2OS (Fig. 5a) and SJSA (Figure S4A) cells. However, DEPTOR expression was dramatically enhanced at both protein (Fig. 5a and S4A) and mRNA (Fig. 5b and S4B) levels only by doxorubicin or nutlin-3 treatment. Next, we silenced p53 to examine whether doxorubicin treatment increased DEPTOR levels by activating p53. Indeed, siRNA-mediated knockdown of p53 by two different targeting sequences abrogated the doxorubicin-induced increase in DEPTOR expression (Fig. 5c, S4C and S4D), suggesting that doxorubicin induces DEPTOR expression in a p53-dependent manner. Moreover, siRNA-mediated p53 knockdown also suppressed DEPTOR expression induced by nutlin-3, indicating that nutlin-3 induces DEPTOR expression in a p53-dependent manner as well (Figure S4E).

It is well known that, as an inhibitor of topoisomerase II, doxorubicin is a wide-spectrum antitumor chemotherapeutic agent that induces DNA damage and apoptosis ${ }^{20}$. Hence, we next attempted to determine whether p53mediated DEPTOR expression affects cancer cell sensitivity to doxorubicin. Indeed, U2OS $\triangle \mathrm{C}-D E P T O R$ cells were more sensitive to doxorubicin, with an $\mathrm{IC}_{50}$ of $98.9 \mathrm{nM}$, which was nearly half of the $\mathrm{IC}_{50}$ value for U2OS WTDEPTOR cells $\left(\mathrm{IC}_{50}=198.6 \mathrm{nM}\right.$, Fig. 6a). Likewise, depletion of site $\mathrm{C}$ in the DEPTOR promoter sensitized cells to low doses of doxorubicin, as reflected by the more dramatic reduction of colony formation upon doxorubicin treatment in $\triangle \mathrm{C}-D E P T O R$ cells (Fig. 6b). Mechanistically, disruption of p53-mediated DEPTOR expression significantly 

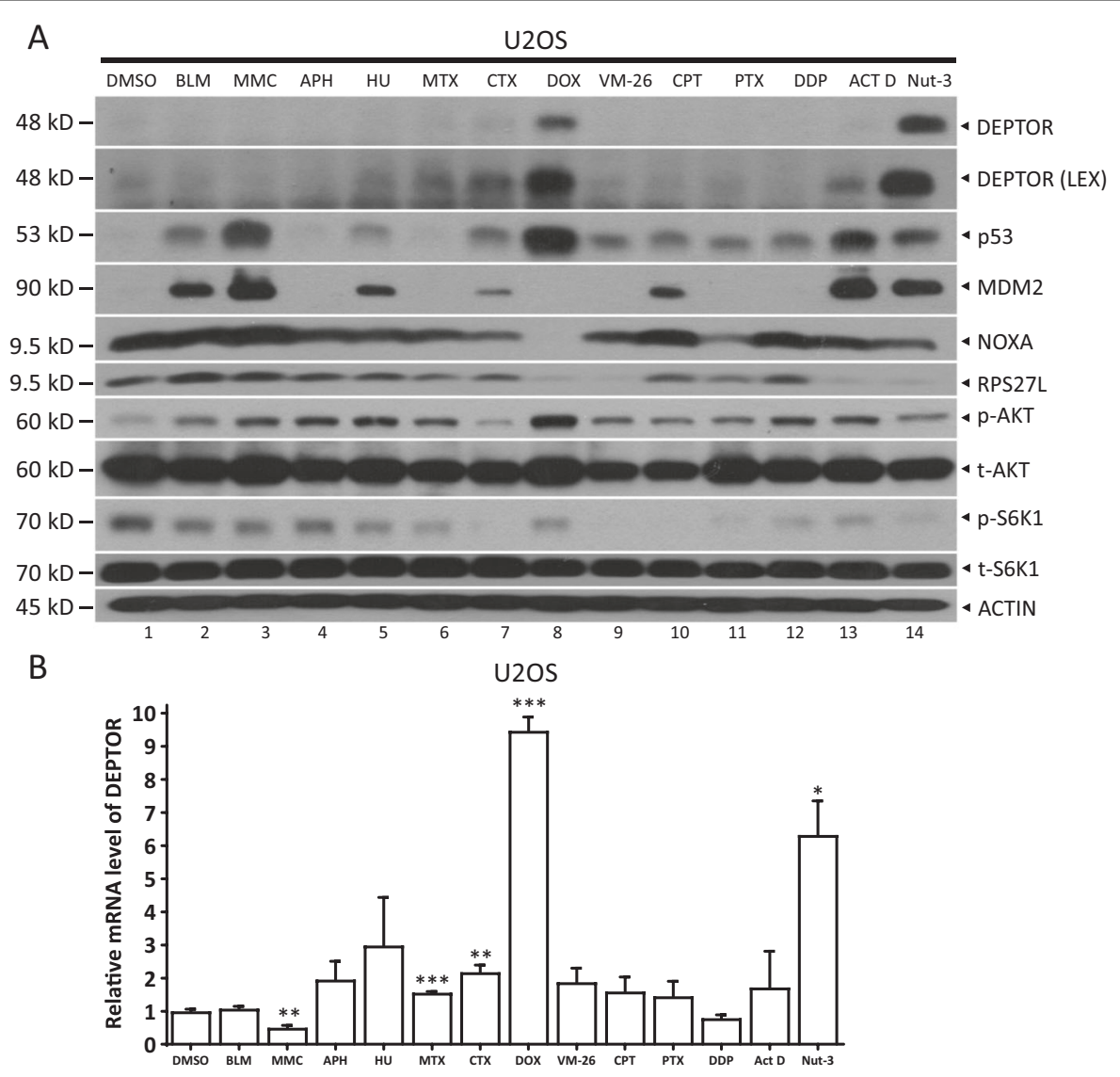

U2OS

C

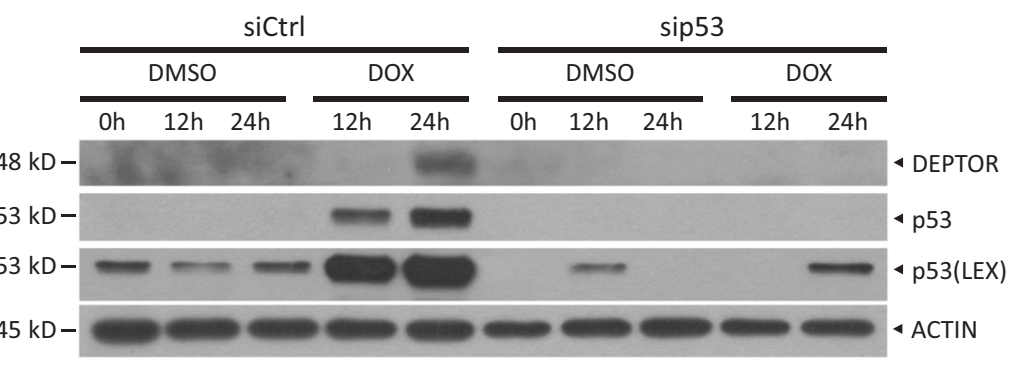

Fig. 5 Doxorubicin treatment induces DEPTOR expression via p53. a, b Doxorubicin treatment dramatically induced DEPTOR expression at both protein and mRNA levels. U2OS cells were treated with various genotoxic agents for $24 \mathrm{~h}$ and then harvested for IB with the indicated Abs a or qRTPCR analysis $\mathbf{b}$ (mean \pm S.E.M, $n=3 ;{ }^{*} p<0.05,{ }^{* *} p<0.01$, ${ }^{* *} p<0.001$, compared with cells treated with DMSO). BLM bleomycin, MMC mitomycin C, APH aphidicolin, HU hydroxyurea, MTX methotrexate, CTX cyclophosphamide, DOX doxorubicin, VM-26 teniposide, CPT camptothecin, PTX paclitaxel, DDP cisplatin, Act D actinomycin D, and Nut-3 nutlin-3. c The induction of DEPTOR upon doxorubicin treatment was dependent on p53. U2OS cells were transfected with the indicated siRNAs for $48 \mathrm{~h}$ and then treated with doxorubicin $(1 \mu \mathrm{M})$ for the indicated time periods, followed by IB with the indicated Abs. LEX longer exposure.

increased doxorubicin-induced apoptosis in a timedependent manner, as evidenced by increased cleavage of PARP and caspase-3 in $\triangle \mathrm{C}-D E P T O R$ cells (Fig. 6c). Besides, although the basal levels of AKT phosphorylation were increased (lanes 5 vs 1) upon DEPTOR-C site depletion, doxorubicin treatment caused a minor induction of AKT phosphorylation (lanes 8 vs 5) in $\triangle \mathrm{C}-D E P T O R$ cells, but a significant induction of AKT phosphorylation in WTDEPTOR cells (lanes 4 vs 1 ), by the relief of feedback inhibition to PI3K (Fig. 6c), which may explain the different doxorubicin sensitivities of these cells. Taken together, our results supported the notion that $\mathrm{p} 53$, activated by doxorubicin treatment, induced DEPTOR expression to activate $\mathrm{AKT}$, thus leading to drug resistance. 


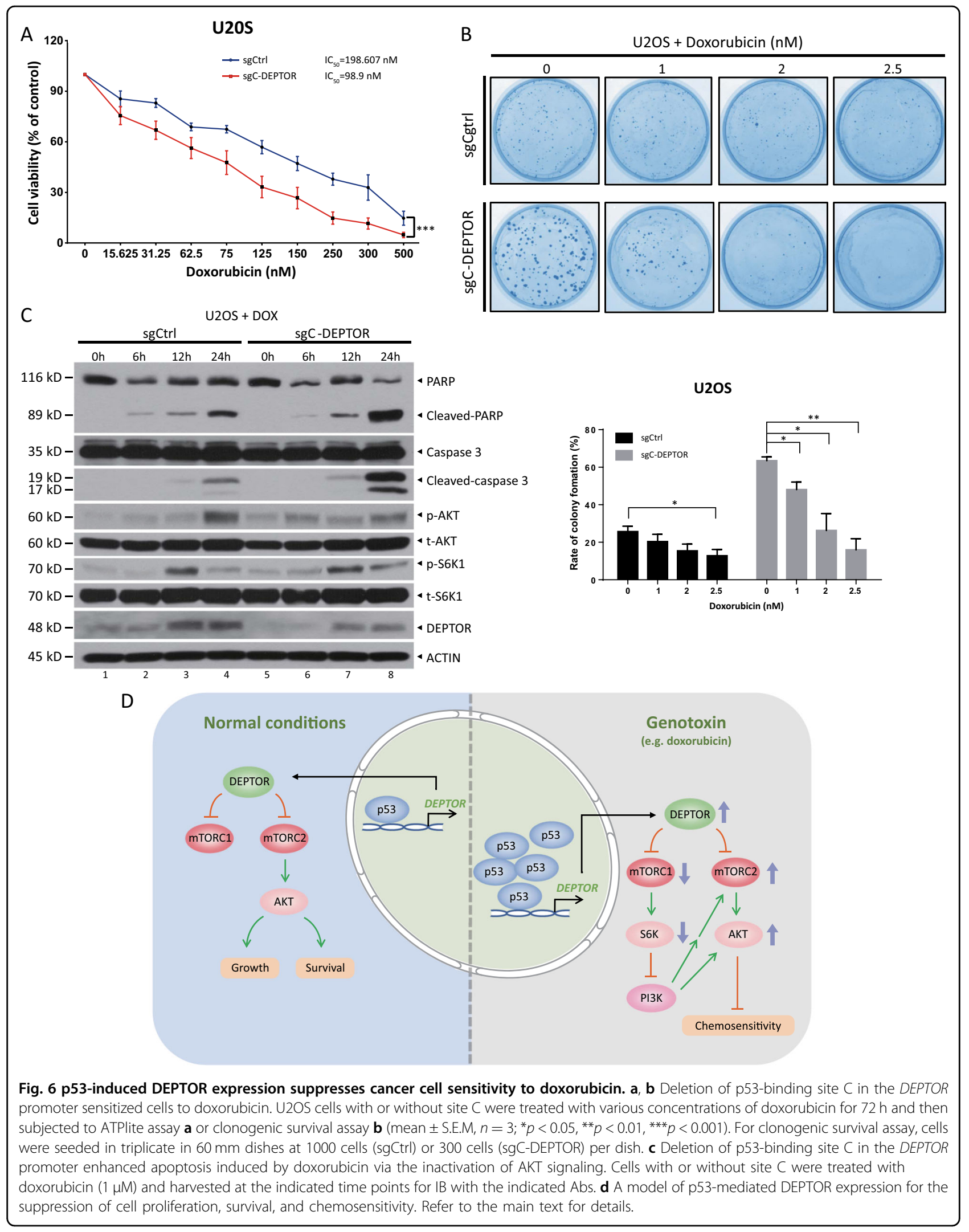




\section{Discussion}

Over the past few years, DEPTOR, a natural inhibitor of mTOR, has been shown to be an important regulator of various physiological and pathological processes, including cell growth and survival, metabolism, immunity, adipogenesis, and tumorigenesis ${ }^{7}$. Here, we identified and characterized DEPTOR as a novel, direct downstream target of the tumor suppressor, p53, based on the following lines of evidence: (1) the protein and mRNA levels of DEPTOR were positively correlated with p53 activity in both cultured cancer cells and mouse tissues; (2) a typical p53 consensus binding site $(-196 \sim-169$, C-DEPTOR $)$ was identified in the DEPTOR promoter; and (3) p53 bound to C-DEPTOR and activated DEPTOR transcription, as determined by dual-luciferase reporter and ChIP assays. Interestingly, deletion of site $\mathrm{C}$ moderately reduced the activity of DEPTOR promoter in sgp53 cells, suggesting that site $C$ may also be regulated by other unknown transcription factor(s) besides p53, which warrants future investigations (Figure S1D). Notably, several specific factors have been identified that likely regulate $D E P T O R$ transcription in cell- and context-specific manners. These factors include: (1) Glucocorticoids, which induce DEPTOR expression in a glucocorticoid receptor-dependent manner during adipogenesis ${ }^{21}$; (2) Baf60c-Six4, which promotes DEPTOR transcription to improve glucose homeostasis in skeletal muscles ${ }^{22}$; and (3) NOTCH1, which activates DEPTOR transcription in T-cell leukemogenesis ${ }^{23}$. However, in our study, we revealed the transcriptional regulation of DEPTOR by the tumor suppressor, p53, in various types of cancer cells, including breast cancer (MCF7), prostate cancer (LNCap), lung cancer (A549), colon cancer (HCT116), and osteosarcoma (U2OS and SJSA) cells, as well as in mouse tissues under unstressed conditions in vivo (Fig. 1 and S1), indicating a key role of p53 in regulating DEPTORmediated physiological processes.

Given that DEPTOR is an inhibitor of both mTOR complexes, we found that p53 regulates mTOR-mediated processes by transactivating DEPTOR expression. We performed knockout of the p53-binding site $\mathrm{C}$ in the $D E P T O R$ promoter $(\triangle \mathrm{C}-D E P T O R)$ in U2OS cells harboring wild-type p53, using CRISPR-Cas9 technology and observed that: (1) $\triangle \mathrm{C}-D E P T O R$ cells proliferate more rapidly than WT-DEPTOR cells (Fig. 3b), (2) $\triangle \mathrm{C}-D E P$ TOR cells have greater survival ability (Fig. 3c), and (3) cell cycle progression is accelerated in $\triangle \mathrm{C}-D E P T O R$ cells (Fig. 3d, e). Mechanistically, disturbing p53-targeted DEPTOR expression activated AKT to promote cell growth and survival (Fig. 4), suggesting that p53 may suppress mTOR signaling by transactivating DEPTOR expression, leading to the inhibition of cell proliferation and survival. Consistently, several upstream negative regulators of mTORC1, including PTEN, TSC2,
AMPK $\beta 1$, and REDD1, are induced by $\mathrm{p} 53$ upon DNA damage ${ }^{19,24-26}$. In this study, we demonstrated that p53 repressed mTORC2 activity, as reflected by the decrease in phosphorylation of AKT at Ser 473, a well-known mTORC2 substrate (Fig. 4a), by inducing DEPTOR expression. Thus, our finding that p53-induced DEPTOR expression to regulate mTORC2 activity added another layer of complexity to the crosstalk between p53 and mTOR pathways.

The tumor suppressor, p53, whose transcriptional activity is significantly increased upon various stresses, has a key role in coordinating DNA damage responses ${ }^{27}$. In this study, we demonstrated that p53 also activates DEPTOR expression in response to genotoxic treatment. We observed that, although various genotoxic agents obviously increased the protein levels of p53, only doxorubicin had significant effects on DEPTOR expression (Fig. 5a, b, S4A and S4B). Likewise, the expression of the three well-established p53 downstream targets, MDM2 ${ }^{12}$, NOXA $^{14}$, and RPS27L $\mathrm{L}^{15,16}$, was not significantly induced by most of the genotoxic agents (Fig. 5a and S4A). These results may be owing to post-translational modifications of p53, such as acetylation, which have been shown to play important roles in transactivating different p53 downstream target genes ${ }^{28,29}$. Thus, it would be interesting to explore whether and how doxorubicin induces the acetylation of p53 at specific lysine residue(s) and in turn, induces the p53-mediated increase in DEPTOR expression. In addition, we found that p53 upregulated DEPTOR expression in a cell density-dependent manner, whereas the protein levels of p53 were not increased (Figure S2). Although the mechanism by which p53 induces DEPTOR expression in response to cell density stress is unknown, it is worth exploring whether acetylation at specific lysine residue(s) regulates p53 activity in a cell densitydependent manner.

Given the negative feedback loop from S6K1 to IRS1/ PI3K-AKT signaling, DEPTOR possesses both tumor suppressive and oncogenic properties in certain contexts ${ }^{5}$. In our study, we demonstrated that, by activating DEPTOR expression, $\mathrm{p} 53$ has distinct roles in regulating AKT activity under unstressed and genotoxic stress conditions. In unstressed cells, p53-mediated DEPTOR expression suppressed the activity of mTOR and its downstream substrate, AKT, leading to the inhibition of cell proliferation and survival (Figs. 3 and 4). However, upon doxorubicin-induced DNA damage, the dramatic induction of DEPTOR expression via p53 hyperactivation alleviated feedback inhibition from S6K1 to IRS1, thereby activating AKT, resulting in cell resistance to doxorubicin (Figs. 5 and 6). Hence, our results suggested that DEPTOR may act as a tumor suppressor at the initiation stage of tumorigenesis, when cells express normal levels of DEPTOR; however, DEPTOR, whose expression is 
significantly enhanced by stresses or anticancer drug treatments, exhibits oncogenic properties to promote cancer cell survival and render the cells resistant to chemotherapeutic drugs ${ }^{30,31}$.

In summary, our study revealed a previously unknown interplay between the tumor suppressor, p53 and the oncoprotein, mTOR, via DEPTOR. Specifically, p53 directly bound to and activated the transcription of DEPTOR, a direct inhibitor of mTOR, to inhibit cell proliferation and survival by repressing $\mathrm{mTORC} 2$ activity in unstressed cells. However, in response to treatment with genotoxic agents, such as doxorubicin, DEPTOR expression was dramatically enhanced by activated p53, leading to cancer cell resistance to doxorubicin by relieving feedback inhibition from S6K1 to IRS1, thus activating AKT. Therefore, DEPTOR is a novel p53 target and p53-mediated DEPTOR expression suppresses cancer cell proliferation, survival, and chemosensitivity to doxorubicin (Fig. 6d).

\section{Methods}

\section{Cell lines and chemicals}

U2OS, SJSA, MCF7, SK-BR3, MDA-MB-231, ZR75-1, and A549 cells were obtained from the American Type Culture Collection (ATCC) and maintained in Dulbecco's modified Eagle's medium (DMEM) supplemented with $10 \%(\mathrm{v} / \mathrm{v})$ fetal bovine serum (FBS). HCT116 $p 53^{+/+}$and $p 53^{-/-}$cells were kindly provided by Professor Bert Vogelstein and maintained in McCoy's $5 \mathrm{~A}$ medium containing 10\% FBS. LNCap, DU145, and PC3 cells were obtained from ATCC and maintained in RPMI-1640 medium containing 10\% FBS. The following chemicals were obtained from commercial sources: bleomycin (HY17565; MCE, Monmouth Junction, NJ, USA), mitomycin C (HY-13316; MCE), hydroxyurea (HY-B0313; MCE), methotrexate (HY-14519; MCE), cyclophosphamide (HY17420; MCE), teniposide (HY-13761; MCE), nutlin-3 (HY-50696; MCE), aphidicolin (sc-201535A; Santa Cruz Biotechnology, Dallas, TX, USA), doxorubicin (S1208; Selleck, Houston, TX, USA), camptothecin (S1288; Selleck), paclitaxel (S1150; Selleck), cisplatin (P4394; SigmaAldrich, St Louis, MO, USA), and actinomycin D (129935; Sigma-Aldrich).

\section{CRISPR/Cas9-based DEPTOR site C and p53 knockout}

Single-guide RNA (sgRNA) was subcloned into the plasmid, pSpCas9(BB)-2A-Puro (PX459). U2OS and SJSA cells were transfected with a sequence-verified CRISPR plasmid and selected with puromycin for 3 days. Single clones were picked under a microscope and confirmed by sequencing and immunoblotting. The sequences of the sgRNA were used as follows: sgC-DEPTOR: 5'-CAAGC TCAAGTTTCTGGGGC-3'; sgp53: 5'-GCAGTCACAGC ACATGACGG-3'.

\section{siRNA silencing}

Cells were transfected with the indicated siRNA oligonucleotides in $60 \mathrm{~mm}$ dishes using Lipofectamine 2000, according to the manufacturer's instructions (Invitrogen, Carlsbad, CA, USA). siCtrl:5'-ATTGTATGCGATCGC AGAC-3'. sip53: 5'-CACCATCCACTACAACTACAT-3'. sip53-2: 5'GCACAGAGGAAGAGAATCT-3'. siAKT: 5'-GAGTTTGAGTACCTGAAGCTG-3'.

\section{ATPlite-based cell proliferation assay}

Cells were seeded in triplicate in 96-well plates $\left(2 \times 10^{3}\right.$ per well). Cell proliferation was evaluated by the ATPlite assay, according to the manufacturer's instructions (PerkinElmer, Waltham, MA, USA). Results are expressed as the fold change compared with the control. To calculate $\mathrm{IC}_{50}$ values, cells were cultured for 3 days with the indicated concentrations of doxorubicin and then subjected to the ATPlite assay.

\section{Clonogenic survival assay}

A total of 300 cells seeded in $60 \mathrm{~mm}$ dishes were grown for 14 days and then stained with Coomassie brilliant blue. The dishes were photographed for colony counting (50 or more cells in a colony). The rate of colony formation (\%) was calculated as colony number/300 $\times 100 \%$.

\section{Dual-luciferase reporter assay}

Cells were co-transfected with pGL3 or pGL3 containing the DEPTOR promoter, along with a plasmid expressing Renilla luciferase. After $24 \mathrm{~h}$, cells were harvested and lysed for the determination of luciferase activity, using a luciferase assay kit (Promega, Madison, WI, USA), according to the manufacturer's instructions. Relative luciferase activity was normalized to Renilla luciferase activity.

\section{ChIP}

ChIP was performed using the SimpleChIP® Enzymatic Chromatin IP Kit (Magnetic Beads; \#9003; Cell Signaling Technology, Danvers, MA, USA), following the manufacturer's instructions. After reverse cross-linking and DNA purification, the immunoprecipitated DNA was amplified by PCR using the following primers: DEPTOR-C-F: 5'-CGCCCAGATGTTTATATTTTCCT-3'; DEPTOR-C-R: 5'-TGCCCTCACAGACGCTTCC-3'; DEPTOR-AB-F: 5'-AATTTTACAAAAGAGGGACAGCAA-3'; DEPTORAB-R: 5'-CAAGGAGAAAAGGGCACTCATAT-3'; MDM2F: 5'-GGTTGACTCAGCTTTTCCTCTTG-3'; MDM2-R: 5'-GGAAAATGCATGGTTTAAAT-3'.

\section{Quantitative reverse transcription PCR}

Total RNA was isolated using TRIzol reagent (Invitrogen) and then reverse transcribed into cDNA using the PrimeScript $^{\mathrm{TM}}$ RT reagent kit (Perfect Real Time; Takara 
Biotechnology, Kusatsu, Japan) following the manufacturer's instructions. Expression levels were measured by quantitative PCR using the SYBR $®$ Premix Ex Taq ${ }^{\mathrm{TM}}$ kit (Tli RNaseH Plus; Takara Biotechnology), according to the manufacturer's instructions. The primers sequences were used as follows: hDEPTOR-F:5'-GCAGCAGGA ATGAAGGTCTG-3', and hDEPTOR-R:5'-GTATGTGC GGAGAAGACTCGTAT-3' for human DEPTOR; hp53F: 5'-TGGAGAATATTTCACCCTTCAGATC-3', and hp53-R: 5'-TTTTTATGGCGGGAGGTAGACT-3' for human p53; hMDM2-F: 5'-CTCTCAGATGAAGATGA TGAGGTATATC-3', and hMDM2-R: 5'-GTTTTCCA GTTTGGCTTTCTCAG-3' for human MDM2; hp21-F: 5'-GGCAGACCAGCATGACAGATT-3', and hp21-R: $5^{\prime}$-GACTAAGGCAGAAGATGTAGAGCG-3' for human p21; hGAPDH-F: 5'-AGGGCATCCTGGGCTACAC-3', and hGAPDH-R: 5'-GCCAAATTCGTTGTCATACC AG-3' for human GAPDH; mDEPTOR-F:5'-GCTGCA GGGATGAAGGTCTG-3', and mDEPTOR-R:5'-CAAA CAGCGTATGAAAGACAAGGT- $3^{\prime}$ for mouse DEPTOR; mp53-F: 5'-GAGAGTATTTCACCCTCAAGATC CG-3', and mp53-R: 5'-CCCCACTTTCTTGACCATT GTTT-3' for mouse p53; mMDM2-F: 5'-GATGA GGATGATGAGGTCTATCGG-3', and mMDM2-R: $5^{\prime}$-TCTGGAAGCCAGTTCTCACGAA- $3^{\prime}$ for mouse MDM2; mGAPDH-F: 5'-GCCGCCTGGAGAAACCTGCC$3^{\prime}$, and mGAPDH-R: 5'-GGTGGAAGAGTGGGAGTTGC$3^{\prime}$ for mouse GAPDH.

\section{Immunoblotting}

Cells were harvested, lysed, and subjected to immunoblotting, as previously described ${ }^{32}$. About $30-60 \mu \mathrm{g}$ of protein were loaded on sodium dodecyl sulfate polyacrylamide gel electrophoresis gels, and the bands with appropriate exposure time are presented. Antibodies against the following proteins were used: DEPTOR (11816, for human samples; Cell Signaling Technology, Danvers, MA, USA; 1:1000), DEPTOR (09-463, for mouse samples; Millipore, Burlington, MA, USA; 1:1000), p53 (32532, for mouse samples; Cell Signaling Technology; 1:1000), p-AKT (S473) (4060; Cell Signaling Technology; 1:2000), t-AKT (4691; Cell Signaling Technology; 1:1000), p-S6K1 (T389) (9234; Cell Signaling Technology; 1:1000), PARP (9532; Cell Signaling Technology; 1:1000), caspase3 (9665; Cell Signaling Technology; 1:1000), cyclin A2 (4656; Cell Signaling Technology; 1:1000), cyclin E2 (4132; Cell Signaling Technology; 1:1000), cyclin B1 (12231; Cell Signaling Technology; 1:1000), cyclin D1 (2978; Cell Signaling Technology; 1:1000), p-H3 (3377; Cell Signaling Technology; 1:1000), t-S6K1 (sc-230; Santa Cruz Biotechnology; 1:1000), NOXA (OP180; Calbiochem, San Diego, CA, USA; 1:1000), MDM2 (OP46; Calbiochem; 1:500), p53 (OP43, for human samples;
Calbiochem; 1:1000), p21 (sc-6246; Santa Cruz Biotechnology; 1:500), actin (ET1701-80; HuaAn Biotechnology, Hangzhou, China; 1:10,000).

\section{Flow cytometry}

Cells were treated with $2 \mathrm{mM}$ thymidine (T1895; SigmaAldrich, St Louis, MO, USA) for $14 \mathrm{~h}$, followed by $9 \mathrm{~h}$ of release. Next, cells were treated with thymidine for another $14 \mathrm{~h}$ and then incubated in fresh medium for different time periods. Cells were harvested and fixed in ice-cold $70 \%$ ethanol overnight. Cells were then stained with PI staining buffer (BD Pharmingen, San Jose, CA, USA) and subjected to flow cytometry.

\section{Statistical analysis}

Statistical analyses were performed by two-tailed Student's $t$ tests, using Statistical Program for Social Sciences software 20.0 (SPSS, Chicago, IL, USA). Data are expressed as mean \pm standard error of the mean (S.E.M). The statistical significance level was set at $p<0.05$.

\section{Acknowledgements}

This work was supported by the National Key R\&D Program of China (2016YFA0501800 to Y.Z. and X.X.), the National Natural Science Foundation of China $(81672728,81972591,92053117$, and 81721091 to Y.Z., 82002924 to D.C., 81572708 , and 81974429 to X.X.), and the Natural Science Foundation of Zhejiang Province (LR16C050001 to Y.Z.).

\section{Author details}

${ }^{1}$ Key Laboratory of Combined Multi-Organ Transplantation, Ministry of Public Health, the First Affiliated Hospital, Zhejiang University School of Medicine, Hangzhou, China. ${ }^{2}$ Institute of Translational Medicine, Zhejiang University School of Medicine, Hangzhou, China. ${ }^{3}$ Cancer Institute of the Second Affiliated Hospital, Zhejiang University School of Medicine, Hangzhou, China

\section{Author contributions}

D.C. and X.D. designed and performed the experiments, analyzed and interpreted the data, and drafted the manuscript. L.G. performed the experiments and revised the manuscript. X.C. and L.W. performed the experiments. X.X. analyzed and interpreted the data, and revised the manuscript. Y.Z. designed the study, analyzed and interpreted the data, and revised and finalized the manuscript. All authors reviewed the manuscript.

\section{Conflict of interest}

The authors declare that they have no conflict of interest.

\section{Publisher's note}

Springer Nature remains neutral with regard to jurisdictional claims in published maps and institutional affiliations.

Supplementary Information accompanies this paper at (https://doi.org/ 10.1038/s41419-020-03185-3).

Received: 1 March 2020 Revised: 27 October 2020 Accepted: 28 October 2020

Published online: 12 November 2020

\footnotetext{
References

1. Zoncu, R., Efeyan, A. \& Sabatini, D. M. mTOR: from growth signal integration to cancer, diabetes and ageing. Nat. Rev. Mol. Cell Biol. 12, 21-35 (2011).
} 
2. Guertin, D. A. \& Sabatini, D. M. Defining the role of mTOR in cancer. Cancer Cell 12, 9-22 (2007)

3. Jung, C. H. et al. mTOR regulation of autophagy. FEBS Lett. 584, 1287-1295 (2010).

4. Peterson, T. R. et al. DEPTOR is an mTOR inhibitor frequently overexpressed in multiple myeloma cells and required for their survival. Cell 137, 873-886 (2009).

5. Zhao, Y. \& Sun, Y. Targeting the mTOR-DEPTOR pathway by CRL E3 ubiquitin ligases: therapeutic application. Neoplasia 14, 360-367 (2012).

6. Wang, Z. et al. An evolving role for DEPTOR in tumor development and progression. Neoplasia 14, 368-375 (2012).

7. Caron, A., Briscoe, D. M., Richard, D. \& Laplante, M. DEPTOR at the nexus of cancer, metabolism, and immunity. Physiol. Rev. 98, 1765-1803 (2018).

8. Vousden, K. H. \& Prives, C. Blinded by the light: the growing complexity of p53. Cell 137, 413-431 (2009).

9. Kruiswijk, F., Labuschagne, C. F. \& Vousden, K. H. p53 in survival, death and metabolic health: a lifeguard with a licence to kill. Nat. Rev. Mol. Cell Biol. 16, 393-405 (2015).

10. Hafner, A., Bulyk, M. L., Jambhekar, A. \& Lahav, G. The multiple mechanisms that regulate p53 activity and cell fate. Nat. Rev. Mol. Cell Biol. 20, 199-210 (2019).

11. Checler, F. \& Alves da Costa, C. p53 in neurodegenerative diseases and brain cancers. Pharm. Ther. 142, 99-113 (2014).

12. Barak, Y., Juven, T., Haffner, R. \& Oren, M. mdm2 expression is induced by wild type p53 activity. EMBO J. 12, 461-468 (1993).

13. el-Deiry, W. S. et al. WAF1, a potential mediator of p53 tumor suppression. Cell 75, 817-825 (1993).

14. Oda, E. et al. Noxa, a BH3-only member of the $\mathrm{BCl}-2$ family and candidate mediator of p53-induced apoptosis. Science 288, 1053-1058 (2000).

15. He, H. \& Sun, Y. Ribosomal protein S27L is a direct p53 target that regulates apoptosis. Oncogene 26, 2707-2716 (2006).

16. Li, J. et al. Ribosomal protein S27-like, a p53-inducible modulator of cell fate in response to genotoxic stress. Cancer Res. 67, 11317-11326 (2007).

17. Nakano, K. \& Vousden, K. H. PUMA, a novel proapoptotic gene, is induced by p53. Mol. Cell 7, 683-694 (2001).
18. Sengupta, S., Peterson, T. R. \& Sabatini, D. M. Regulation of the mTOR complex 1 pathway by nutrients, growth factors, and stress. Mol. Cell 40, 310-322 (2010).

19. Feng, Z. \& Levine, A. J. The regulation of energy metabolism and the IGF-1/ mTOR pathways by the p53 protein. Trends Cell Biol. 20, 427-434 (2010).

20. Tacar, O., Sriamornsak, P. \& Dass, C. R. Doxorubicin: an update on anticancer molecular action, toxicity and novel drug delivery systems. J. Pharm. Pharm. 65, 157-170 (2013).

21. Laplante, M. et al. DEPTOR cell-autonomously promotes adipogenesis, and its expression is associated with obesity. Cell Metab. 16, 202-212 (2012).

22. Meng, Z.-X. et al. Baf60c drives glycolytic metabolism in the muscle and improves systemic glucose homeostasis through Deptor-mediated Akt activation. Nat. Med 19, 6 (2013).

23. Hu, Y. et al. DEPTOR is a direct NOTCH1 target that promotes cell proliferation and survival in T-cell leukemia. Oncogene 36, 1038-1047 (2017).

24. Stambolic, V. et al. Regulation of PTEN transcription by p53. Mol. Cell 8 , 317-p325 (2001).

25. Ellisen, L. W. et al. REDD1, a developmentally regulated transcriptional target of p63 and p53, links p63 to regulation of reactive oxygen species. Mol. Cell 10, 995-1005 (2002).

26. Feng, Z., Zhang, H., Levine, A. J. \& Jin, S. The coordinate regulation of the p53 and mTOR pathways in cells. Proc. Natl Acad. Sci. USA 102, 8204-8209 (2005).

27. Riley, T., Sontag, E., Chen, P. \& Levine, A. Transcriptional control of human p53regulated genes. Nat. Rev. Mol. Cell Biol. 9, 402-412 (2008).

28. Li, T. et al. Tumor suppression in the absence of p53-mediated cell-cycle arrest, apoptosis, and senescence. Cell 149, 1269-1283 (2012).

29. Wang, S.-J. et al. Acetylation is crucial for p53-mediated ferroptosis and tumor suppression. Cell Rep. 17, 366-373.

30. Zhao, Y., Xiong, X. \& Sun, Y. DEPTOR, an mTOR inhibitor, is a physiological substrate of SCF $\beta$ TrCP E3 ubiquitin ligase and regulates survival and autophagy. Mol. Cell 44, 304-316 (2011).

31. Gao, D. et al. mTOR drives its own activation via SCF $\beta$ TrCP-dependent degradation of the mTOR Inhibitor DEPTOR. Mol. Cell 44, 290-303 (2011).

32. Cui, D. et al. The cross talk of two family members of beta-TrCP in the regulation of cell autophagy and growth. Cell Death Differ. 27, 1119-1133 (2020). 